\title{
One-minute Joule Annealing Enhances the Thermoelectric Properties of Carbon Nanotube Yarns via the Formation of Graphene at the Interface
}

\section{Supporting Information}

\begin{abstract}
Masaki Hada, ${ }^{1,2 *}$ Taisuke Hasegawa, ${ }^{3 \dagger}$ Hirotaka Inoue, ${ }^{1+}$ Makito Takagi, ${ }^{4}$ Kazuki Omoto, ${ }^{1}$ Daiki Chujo, ${ }^{1}$ Shogo Iemoto, ${ }^{1}$ Taihei Kuroda,${ }^{1}$ Taiga Morimoto, ${ }^{1}$

Takuma Hayashi, ${ }^{1}$ Toru Iijima, ${ }^{1}$ Tomoharu Tokunaga, ${ }^{5}$ Naoshi Ikeda, ${ }^{1}$ Kazuhiro Fujimori, ${ }^{1}$ Chihiro Itoh, ${ }^{6}$ Takeshi Nishikawa, ${ }^{1}$ Yoshifumi Yamashita, ${ }^{1}$ Toshihiko Kiwa, ${ }^{7}$ Shin-ya Koshihara, ${ }^{8}$ Satoshi Maeda, ${ }^{9,10,11^{*}}$ and Yasuhiko Hayashi ${ }^{1 *}$

${ }^{1}$ Graduate School of Natural Science and Technology, Okayama University, Okayama 700-8530, Japan. ${ }^{2}$ Tsukuba Research Center for Interdisciplinary Materials Science (TREMS), Faculty of Pure and Applied Sciences, University of Tsukuba, Tsukuba 3058573, Japan. ${ }^{3}$ Materials Open Platform for Chemical Industry, External Collaboration Division, National Institute for Materials Science, Tsukuba 305-0047, Japan. ${ }^{4}$ Graduate School of Chemical Sciences and Engineering, Hokkaido University, Sapporo 060-8628, Japan. ${ }^{5}$ Graduate School of Engineering, Nagoya University, Nagoya 464-8603, Japan. ${ }^{6}$ Faculty of System Engineering, Wakayama University, Wakayama 640-8510, Japan. ${ }^{7}$ Graduate School of Interdisciplinary Science and Engineering in Health Systems, Okayama University, Okayama 700-8530, Japan. ${ }^{8}$ Department of Chemistry, Tokyo Institute of Technology, Tokyo 152-8551, Japan. ${ }^{9}$ Institute for Chemical Reaction Design and Discovery (WPI-ICReDD), Hokkaido University, Sapporo 001-0021, Japan. ${ }^{10}$ Department of Chemistry, Faculty of Science, Hokkaido University, Sapporo 060-0810, Japan. ${ }^{11}$ Research and Services Division of Materials Data and Integrated System (MaDIS), National Institute for Materials Science, Tsukuba 305-0044, Japan.
\end{abstract}

†These authors contributed equally to this work.

*E-mails: Masaki Hada (hada.masaki.fm@u.tsukuba.ac.jp)

Satoshi Maeda (smaeda@eis.hokudai.ac.jp)

Yasuhiko Hayashi (hayashi.yasuhiko@okayama-u.ac.jp) 
Table of Contents

1. Theoretical prediction of the interfacial structure of the CNTs (Note S1 and Figures S1-S2)

2. Fabrication and characterization of the CNT yarns (Figure S3-S7)

3. Thermoelectric and mechanical properties of the CNT yarns (Figures S8-S10)

Supporting References 


\section{Theoretical prediction of the interfacial structure of the CNTs}

Note S1

Calculation of the average number of $\mathrm{sp}^{2}$-hybridized bonds

The rate constant matrix contraction (RCMC) method ${ }^{\mathrm{S} 1}$ classifies the local energyminimum structures, the so-called equilibrium structures (EQs), into super states (SSs), which are defined as the states where all the EQs in each SS achieve a Boltzmann distribution for the given timescale at a given temperature. The RCMC method is a fuzzy clustering method, i.e., the responsibility weight, $c_{\alpha i}$, ranges from 0 to 1 . For example, if $c_{\alpha i}=0.5$, half of the $i$ th EQ belongs to the $\alpha$ th SS. The average number of $\mathrm{sp}^{2}-$ hybridized bonds in the $\alpha$ th SS is represented by:

$$
\left\langle N^{s p^{2}}\right\rangle_{S S(\alpha)}=\left(\sum_{i=1}^{N_{E Q}} c_{\alpha i} e^{-\beta E_{i}} N_{i}^{s p^{2}}\right)\left(\sum_{k=1}^{N_{E Q}} c_{\alpha k} e^{-\beta E_{k}}\right)^{-1},
$$

where $N_{E Q}$ is the number of EQs, $\beta$ the inverse temperature, $E_{i}$ the structural energy of the $i$ th EQ, and $N_{i}^{\mathrm{sp}^{2}}$ the number of $\mathrm{sp}^{2}$-hybridized bonds in the structure of the $i$ th EQ. Here, bond lengths in the range of $0.138-0.149 \mathrm{~nm}$ are considered $\mathrm{sp}^{2}$ hybridized bonds. With the given initial population, the populations of the $\alpha$ th SS at the given time, $t, \rho_{\alpha}(t)$, can be calculated by the full (f)-RCMC method. Then, the average number of $\mathrm{sp}^{2}$-hybridized bonds at time $t$ is:

$$
\left\langle N^{s p^{2}}\right\rangle(t)=\sum_{\alpha=1}^{N_{s s}} \rho_{\alpha}(t)\left\langle N^{s p^{2}}\right\rangle_{S S(\alpha)} .
$$

In this study, we have assumed that the population of the initial structure was unity and all others were zero. 


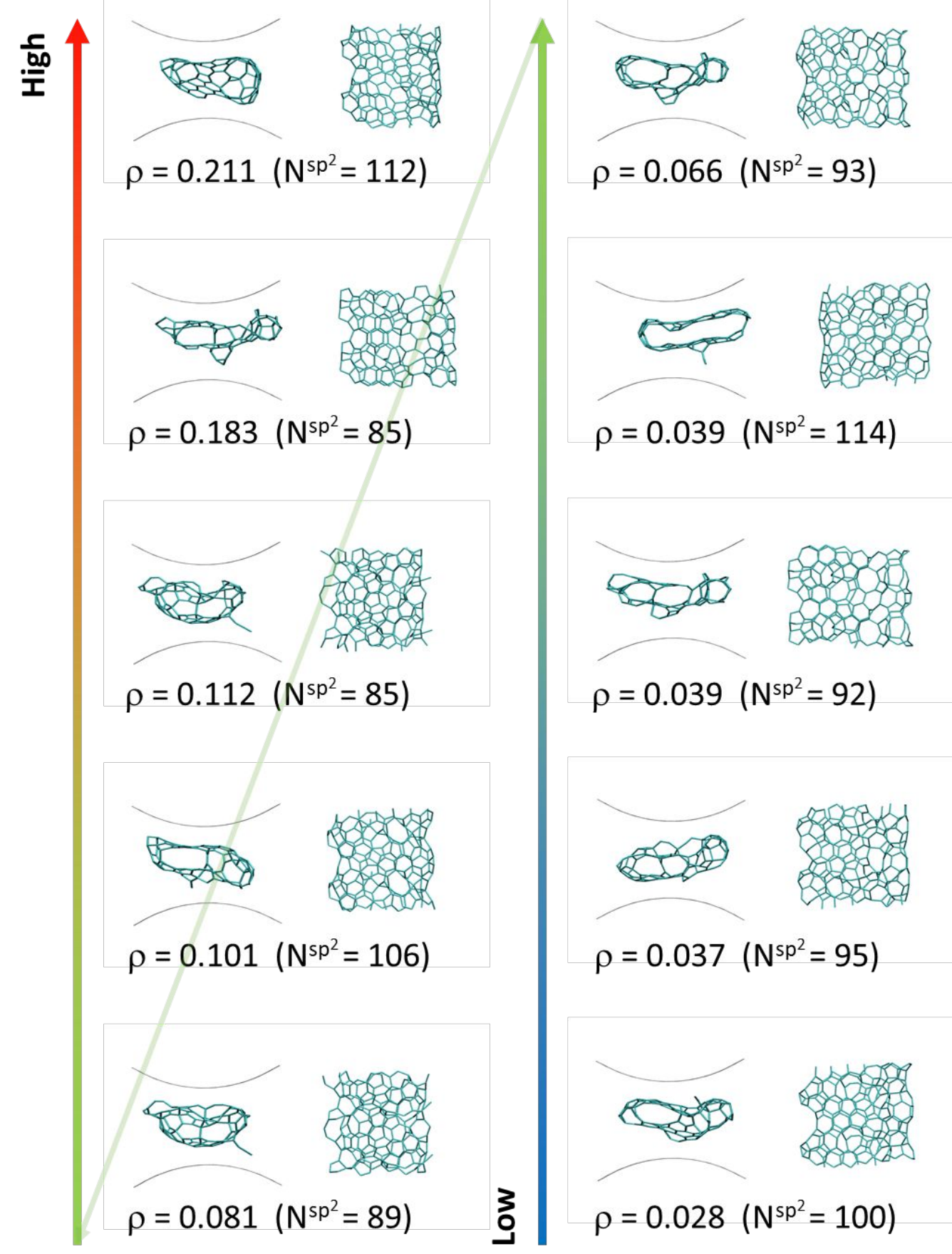

Figure S1. Top 10 most populated local energy-minimum structures after one-minute annealing at $2000 \mathrm{~K}$ predicted by the f-RCMC method. CNTs are shown in line representation, while amorphous carbon atoms are shown in stick representation. The right figures in each column are the top views, while the left figures are the cut views. The unit cell is doubled along the axial direction of the CNTs for clarity. The number of $\mathrm{sp}^{2}$-hybridized bonds is shown in parentheses. The molecular graphics were created using the Visual Molecular Dynamics (VMD) program ${ }^{\mathrm{S} 2}$. 


\section{Fabrication and characterization of the CNT yarns}

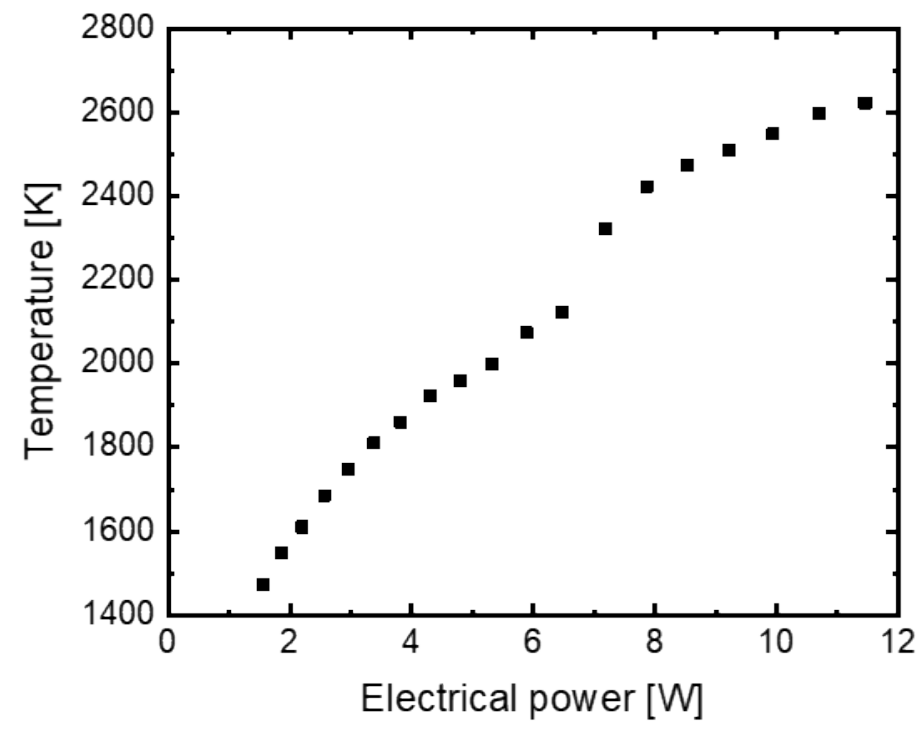

Figure S2. The annealing temperature of a CNT yarn as a function of the applied electrical power. The temperature was measured using a radiation thermometer. The typical applied power was $5 \mathrm{~W}$; therefore, the temperature of the CNT yarn during annealing was $\sim 2000$ K. 


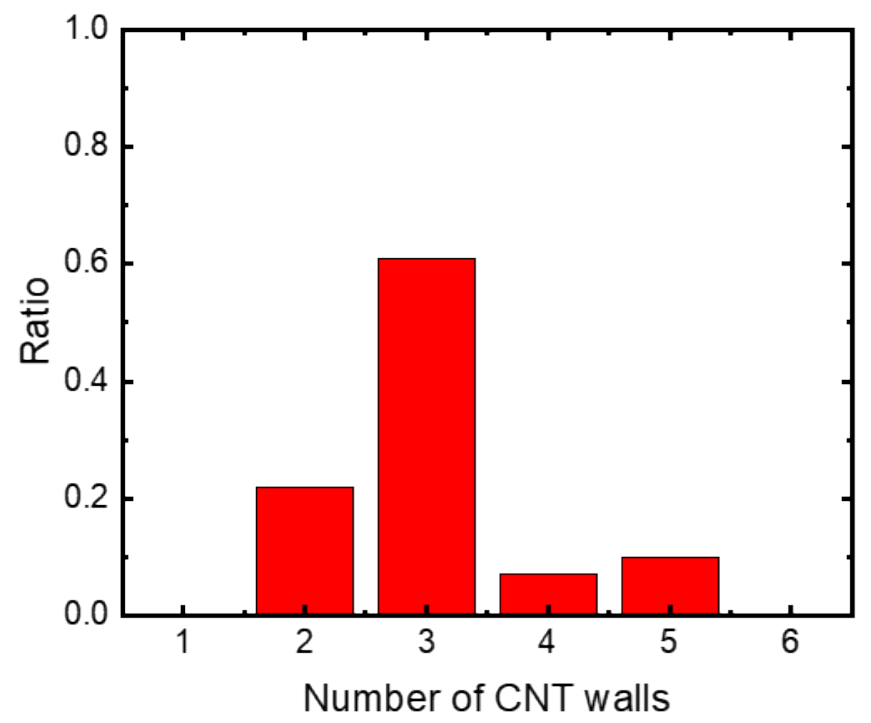

Figure S3. The number of CNT walls obtained from TEM images. One hundred CNTs were evaluated. 


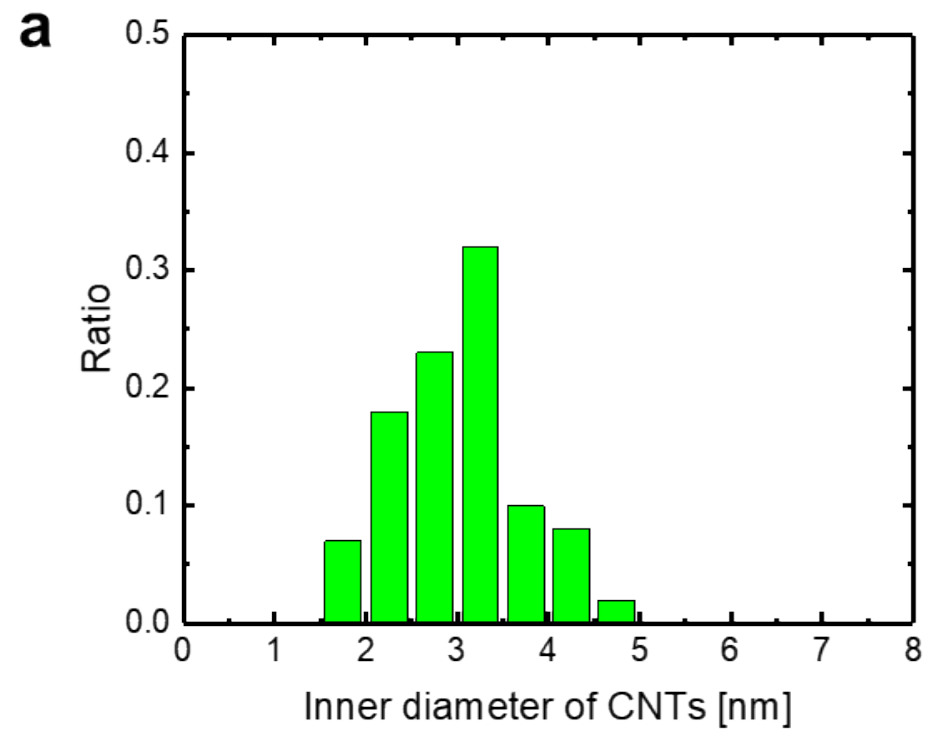

b

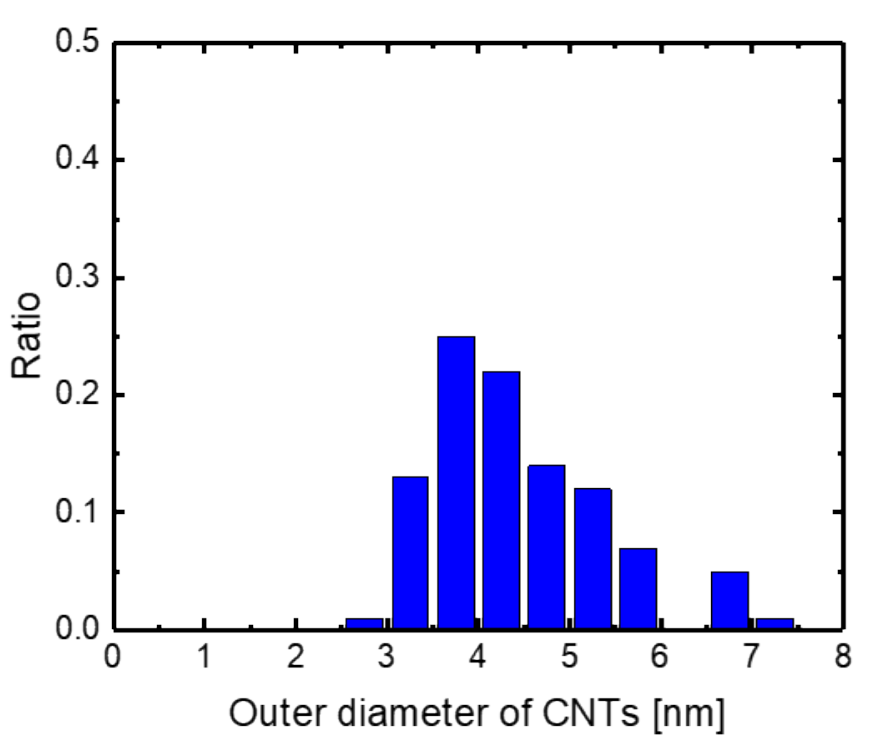

Figure S4. Inner (a) and outer (b) diameter of the CNTs obtained from TEM images. One hundred CNTs were evaluated. 

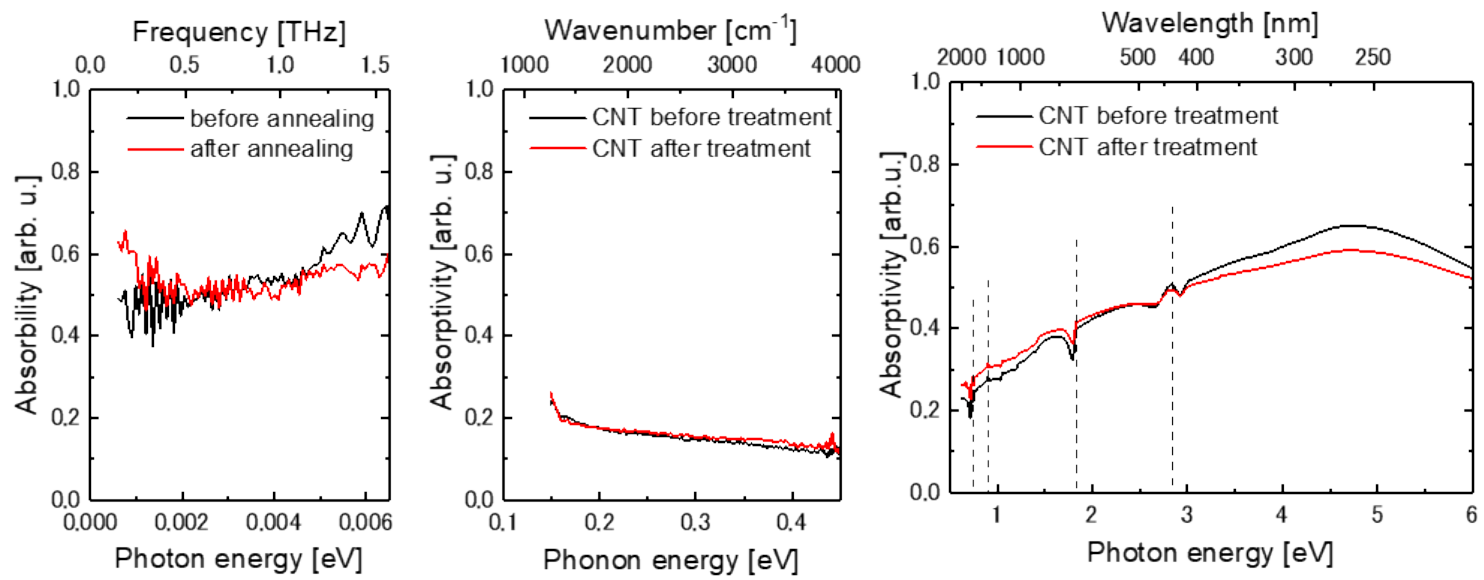

Figure S5. Absorption spectra. Optical transmission spectra of the CNT webs in the terahertz, mid-IR, visible, and ultraviolet regions. The absorption spectra did not change significantly after annealing. 


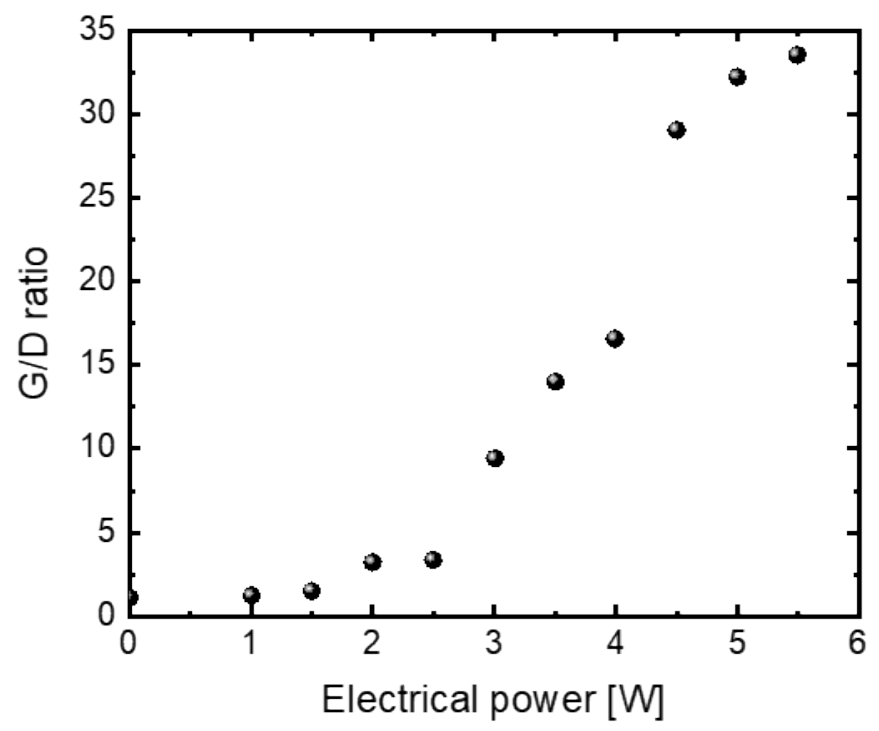

Figure S6. Raman G/D ratio of annealed CNT yarns as a function of the electrical power. 
3. Thermoelectric and mechanical properties of the CNT yarns

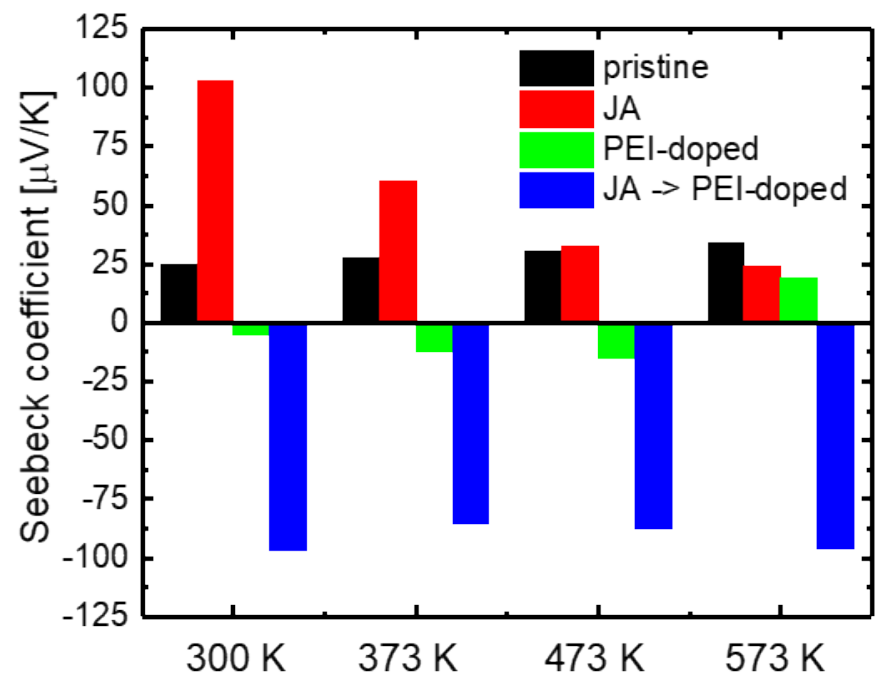

Figure S7. Temperature dependence of the Seebeck coefficients of the pristine, Jouleannealed (JA), PEI-doped, as well as PEI-doped and Joule-annealed CNT yarns. 


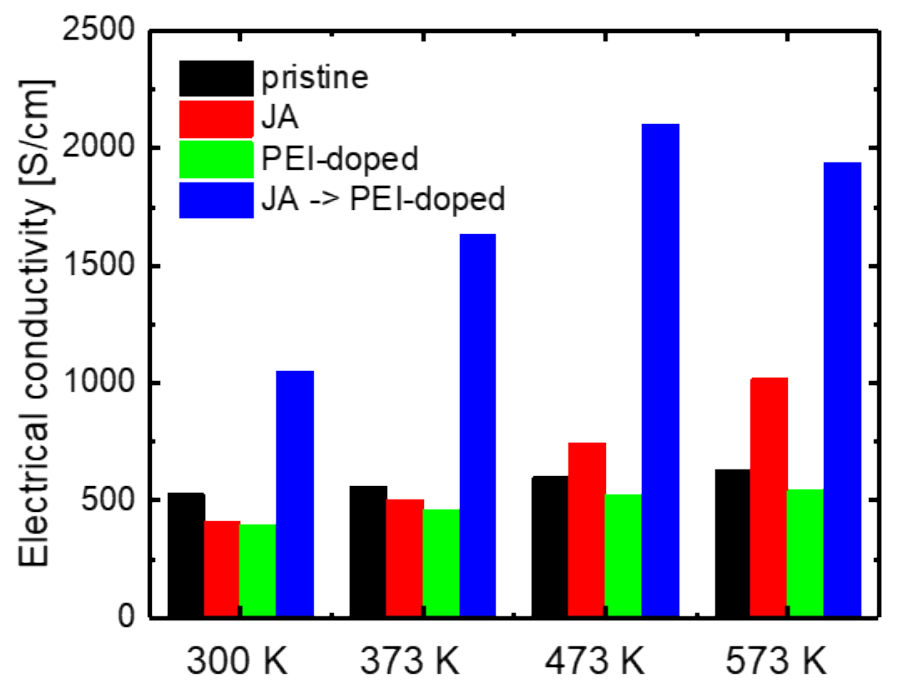

Figure S8. Temperature dependence of the electrical conductivity of the pristine, Jouleannealed (JA), PEI-doped, as well as PEI-doped and Joule-annealed CNT yarns. 


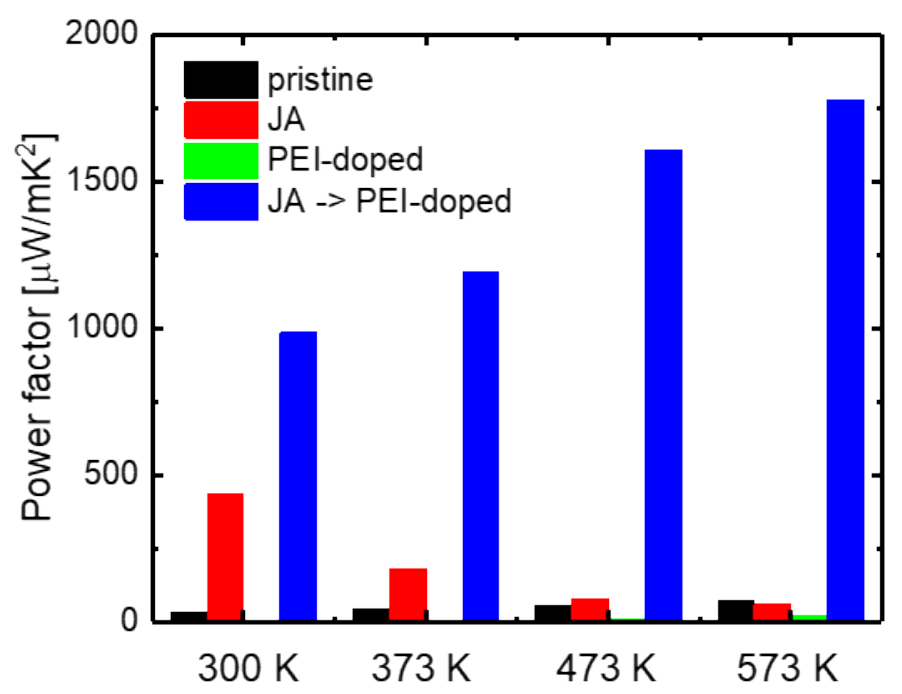

Figure S9. Temperature dependence of the thermoelectric power factors of the pristine, Joule-annealed (JA), PEI-doped, as well as PEI-doped and Joule-annealed CNT yarns. 

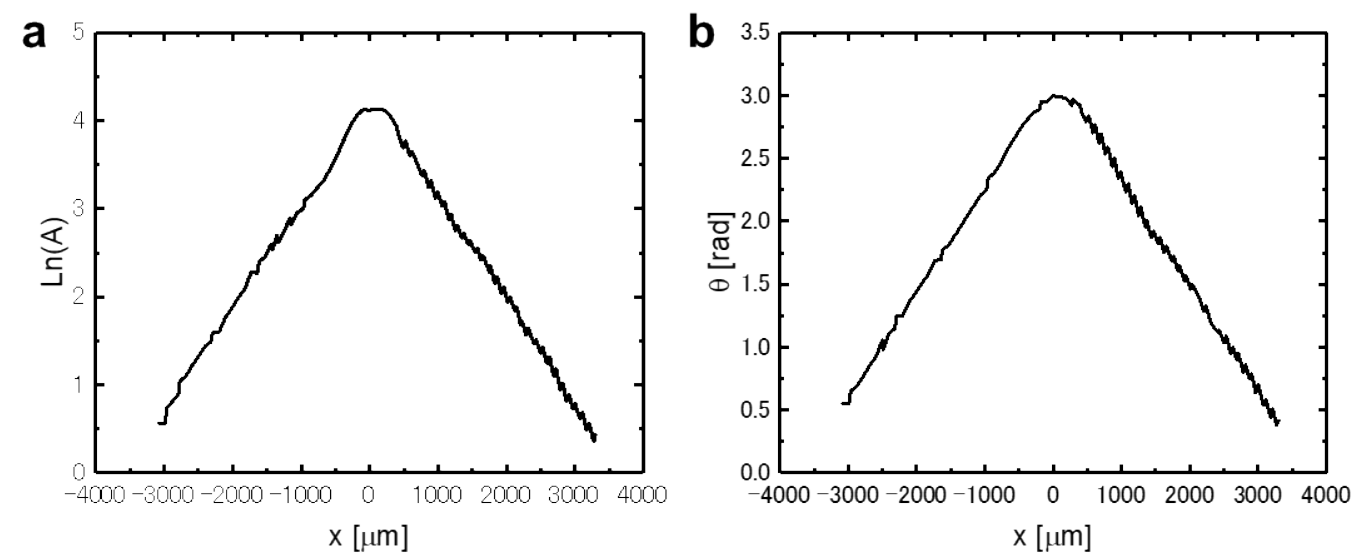

Figure S10. The thermal diffusivity of the CNT yarns measured by modified AC calorimetry. ${ }^{\mathrm{S} 3}$ The thermal diffusivity from amplitude $\left(\alpha_{\mathrm{a}}\right)$ (a) and phase $\left(\alpha_{\mathrm{p}}\right)$ (b) are given by $A \propto \exp \left(-x \sqrt{\omega / 2 \alpha_{\mathrm{a}}}\right)$ and $\theta \propto-x \sqrt{\omega / 2 \alpha_{\mathrm{p}}}$, where $A$ and $\theta$ the amplitude and phase of temperature-modulation measured by a thermocouple, $x$, and $\omega$ are the heated position from the thermocouple and angular frequency $(\omega=2 \pi f, f=10 \mathrm{~Hz})$. The thermal diffusivity $(\alpha)$ is given by $\alpha=\sqrt{\alpha_{\mathrm{a}} \cdot \alpha_{\mathrm{p}}}$. The thermal diffusivity of the CNT yarns was measured to be $3.27 \pm 0.01 \times 10^{-5} \mathrm{~m}^{2} / \mathrm{s}$. Thermal conductivity $(k)$ was calculated with the density $(\rho)$ and specific heat $(c)$ of the material as $\alpha=k / \rho c$. As mentioned in the main text, density and specific heat of the material are $1.0 \mathrm{~g} / \mathrm{cm}^{3}$ and $0.74 \mathrm{~J} / \mathrm{g} \cdot \mathrm{K}$, respectively. ${ }^{\mathrm{S} 4}$ 


\section{Supplementary References}

S1. Sumiya, Y.; Nagahata, Y.; Komatsuzaki, T.; Taketsugu, T.; Maeda, S. Kinetic Analysis for the Multistep Profiles of Organic Reactions: Significance of the Conformational Entropy on the Rate Constants of the Claisen Rearrangement. $J$. Phys. Chem. A 2015, 119, 11641-11649.

S2. Humphrey, W.; Dalke, A.; Schulten, K. VMD: Visual Molecular Dynamics. $J$. Mol. Graph. 1996, 14, 33-38.

S3. Kato, R.; Maesono, A.; Tye, R. P. Thermal Conductivity Measurement of Submicron-Thick Films Deposited on Substrates by Modified ac Calorimetry (Laser-Heating Ångstrom Method). Int. J. Thermophys. 2001, 22, 617-629.

S4. Pradhan, N. R.; Duan, H.; Liang, J.; Iannacchione, G. S. The Specific Heat and Effective Thermal Conductivity of Composites Containing Single-Wall and MultiWall Carbon Nanotubes. Nanotechnology 2009, 20, 245705. 